\title{
Co-channel interference cancellation using precoding for bi-direction multiple hops MIMO relay systems
}

\author{
Vu Van Son, Nguyen Huy Hoang and Pham Thanh Hiep*
}

\begin{abstract}
In order to increase performance of a system while saving power consumption, multiple hop MIMO relay systems (MHMRSs) have been considered. There are many researches on the physical (PHY) layer, medium access control (MAC) layer, and MAC-PHY cross-layer to avoid a co-channel interference, such as the matched/orthogonal weight algorithm, TDMA-based smart antenna and so on. The matched/orthogonal algorithm is available for bi-directional communications; however, it requests at least three antenna elements at every relay. Consequently, it is unavailable for small wireless devices that are being demanded. In this paper, we propose an algorithm to cancel the co-channel interference of bi-directional communication MHMRSs by signal processing at receivers using precoding at transmitters. The proposal algorithm can perform with only two antenna elements while maintaining character of MIMO. The proposal algorithm is compared to the TDMA-based smart antenna algorithm which also can perform with two antenna elements, in both perfect and imperfect channel state information (CSI) schemes. Numerical calculation results indicate that a channel capacity of proposal algorithm is much higher than that of TDMA-based smart antenna algorithm in the perfect CSI scheme due to complete cancellation of co-channel interference and viability of bi-directional communications of proposal algorithm. In the imperfect CSI scheme, the channel capacity of the TDMA-based smart antenna algorithm is more robust when channel estimation errors increase; however, the channel capacity of proposal algorithm is still higher when the channel estimation error is large.
\end{abstract}

Keywords: Co-channel interference cancellation using precoding, Multiple hop MIMO relay system, Channel capacity of system, Perfect and imperfect channel state information, TDMA-based smart antenna

\section{Introduction}

Limitations on frequency resources and transmit powers are important problems for wireless communication networks. A frequency reuse and reduction of transmit powers has been researched, and multiple hop networks are known as an attractive technology. There are many researches on multiple hop technologies in ad hoc systems for both overlay [1] and underlay [2] scenarios. In order to improve performance of multiple hop systems, several control methods on medium access control (MAC) layer have been proposed, i.e, S-MAC [3], T-MAC [4], B-MAC [5], X-MAC [6], Wise-MAC [7], etc. However,

*Correspondence: phamthanhhiep@gmail.com Le Quy Don Technical University, 236 Hoang Quoc Viet, Nghia Do, Cau Giay, 100000 Ha Noi, Viet Nam these researches are effective in low-duty cycle and delaytolerant multiple hop networks. For systems that demand high-duty cycle and/or high channel capacity, another control method is requested.

Multiple input multiple output (MIMO) systems are being applied into many wireless systems to increase the channel capacity. In order to maintain the high channel capacity while saving transmit powers and sharing spectrum, multiple hop MIMO relay systems (MHMRSs) are taken into account. MHMRSs have been analyzed in both decode and forward (DF) [8, 9] and amplify and forward (AF) $[10,11]$ schemes. We have optimized that distances between each transceiver transmit powers of every transmitter to improve the performance of MHMRSs whether there is a control on MAC layer [12, 13] or not [14]. A problem of MHMRSs is the co-channel interference from adjacent relays that let the performance of MHMRSs

\section{Springer}

(c) 2016 Son et al. Open Access This article is distributed under the terms of the Creative Commons Attribution 4.0 International License (http://creativecommons.org/licenses/by/4.0/), which permits unrestricted use, distribution, and reproduction in any medium, provided you give appropriate credit to the original author(s) and the source, provide a link to the Creative Commons license, and indicate if changes were made. 
deteriorate. In order to avoid the co-channel interference, time division multiple access (TDMA), frequency division multiple access (FDMA), code division multiple access (CDMA) and so on have been proposed. According to TDMA, FDMA, or CDMA, the co-channel interference is avoided; however, the channel capacity of multiple hop systems is deteriorated due to considerably small allocation time, small bandwidth, or long length of sequence for every relay $[12,13,15,16]$. There are several researches that use properties of MIMO to cancel the co-channel interference of adjacent relays, such as a dirty paper coding (DPC), zero forcing (ZF) [17], network coding (NC) [18] and so on. Additionally, a smart antenna technology has been proposed, by controlling antennas combining weight to be orthogonal to the interference signal and to be matched to the desired signal, the co-channel interference is reduced, and a diversity gain is achieved. As a result, the channel capacity of systems is improved $[19,20]$. The matched/orthogonal weight algorithm can avoid the co-channel interference and supply link multiplexing simultaneously in a single channel. Every relay can transmit data to bi-direction in the same channel, and the channel capacity is improved by means of networkoriented MIMO interference cancellation that cannot be achieved by TDMA-based smart antenna MIMO networks. However, a relay should design its weights to be orthogonal to two interference signals and to be marched to two desired signals from adjacent relays. Therefore, the relay should have at least three antenna elements, and it is unavailable to small devices, such as mobile phones, vital sensors and so on. Furthermore, the interference from two adjacent relays is cancelled; however, the co-channel interference from other relays still affects the performance of MHMRSs.

In this paper, we propose a method to cancel the cochannel interference from all sensors that share the spectrum while achieving the diversity/multiplexing gains. The proposal algorithm uses the precoding at every transmitter; therefore, it is called as co-channel interference cancellation using precoding (CICP) for multiple hop MIMO relay systems. Similar to the smart antenna technology, in the proposed MHMRS, every relay transmits signals to bi-direction in the same channel. The performance of CICP algorithm is analyzed in case the number of antenna elements is two, the extension to another number of antenna elements is straightforward. However, the matched/orthogonal weight algorithm cannot perform with two antenna elements. Therefore, the proposal algorithm is compared to the TDMA-based smart antenna algorithm that is available to two antenna elements. The performance of proposal algorithm is analyzed in both perfect and imperfect channel state information (CSI) and a validity is evaluated by numerical calculation of ergodic channel capacity.
The notation used in this paper is as follows. Regular and bold styles, respectively, represent a scalar and a vector/matrix. Let $\mathbf{X}^{\mathrm{B}}$ and $\mathbf{X}^{\mathrm{F}}$ denote signal vectors for backward link and forward link, respectively. $\mathbf{X}^{\mathcal{H}}, \mathbf{X}^{\mathrm{T}}$, and $\mathbf{X}^{-1}$ represent the Hermitian, transpose, and pseudo-inverse operations of $\mathbf{X} . E[\cdot]$ describes an expectation operation. Since a component-wise form is adopted to analyze the performance of system, let $\mathbf{X}\{1\}$ denote the $1^{\text {th }}$ row of X. $\mathbb{C}^{2 \times 2}$ describes two rows, two columns normalized matrices from zero mean unit variance independent and identically distributed (i.i.d.) complex Gaussian entries matrices. The normalization operation is to emphasize the signal processing and keep the amplitude of signals from the variation by signal processing.

The rest of the paper is organized as follows. We introduce the system model and analyze the performance of CICP algorithm for MHMRSs in perfect CSI in Section 2. The performance analysis of proposal and TDMA-based smart antenna algorithm in the imperfect CSI is described in Section 3. Section 4 shows the numerical calculation result and Section 5 concludes the paper.

\section{CICP algorithm}

\subsection{System model}

As explained in the previous section, all terminals are equipped with two antenna elements, and all antenna elements are used to transmit or receive signals; therefore, the transmission topology is the half-duplex. However, signals of forward and backward links are transmitted in the same time meaning bi-directional communications. After receiving the signal, relays process the signal to cancel the interference signal and obtain the desired signal, it means that a decode and forward (DF) scheme is applied. The signal processing at $i^{\text {th }}$ relay while $i-1^{\text {th }}$ and $i+1^{\text {th }}$ relays are transmitting is depicted in Fig. 1.

The transmit signal is multiplied by precoding vector before it is transmitted. The precoding vector for backward link and forward link is, respectively, described as $\mathbf{P}^{\mathrm{B}}$ and $\mathbf{P}^{\mathrm{F}}$; therefore, the transmit signal of $\mathrm{i}-1^{\text {th }}$ and $\mathrm{i}+1^{\text {th }}$ relays is represented as follows.

$$
\begin{aligned}
& \mathbf{X}_{\mathrm{i}+1}=\mathbf{P}_{\mathrm{i}+1}^{\mathrm{B}} \mathbf{S}_{\mathrm{i}+1}^{\mathrm{B}}+\mathbf{P}_{\mathrm{i}+1}^{\mathrm{F}} \mathbf{S}_{\mathrm{i}+1}^{\mathrm{F}}, \\
& \mathbf{X}_{\mathrm{i}-1}=\mathbf{P}_{\mathrm{i}-1}^{\mathrm{B}} \mathbf{S}_{\mathrm{i}-1}^{\mathrm{B}}+\mathbf{P}_{\mathrm{i}-1}^{\mathrm{F}} \mathbf{S}_{\mathrm{i}-1}^{\mathrm{F}},
\end{aligned}
$$

here, $\mathbf{S}^{\mathrm{B}}$ and $\mathbf{S}^{\mathrm{F}}$ denote the transmit signal of backward and forward links, respectively. For $\mathrm{i}^{\text {th }}$ relay, $\mathbf{S}_{\mathrm{i}+1}^{\mathrm{B}}$ and $\mathbf{S}_{\mathrm{i}-1}^{\mathrm{F}}$ are the desired signals whereas $\mathbf{S}_{\mathrm{i}+1}^{\mathrm{F}}$ and $\mathbf{S}_{\mathrm{i}-1}^{\mathrm{B}}$ are the interference signals. In fact, the $\mathrm{i}^{\text {th }}$ relay receives the interference signal from not only adjacent relays but also all relays that transmit signals in the same time. The sum of interference signals to $\mathrm{i}^{\text {th }}$ relay is denoted by $\boldsymbol{S}_{\mathrm{i}}^{\text {int }}$. In order to depict the signal processing at $\mathrm{i}^{\text {th }}$ relay intelligibly, the signal that is received from $y_{1}$ and $y_{2}$ antenna elements is represented by the solid line and the broken 

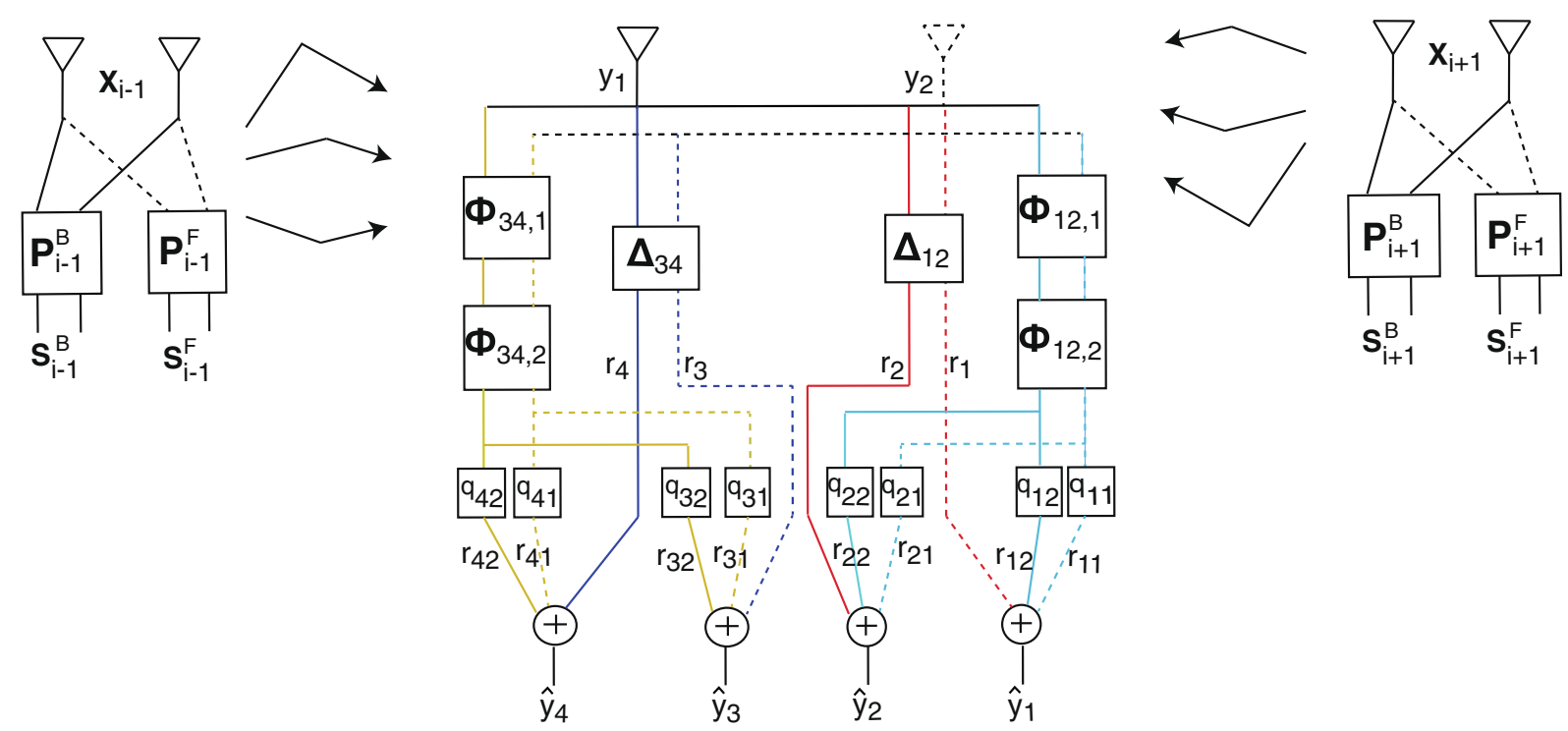

Fig. 1 The system model of CICP algorithm for MHMRSs

line, respectively. Furthermore, signals from the different signal processing are described by the different color. The received signal of $\mathrm{i}^{\text {th }}$ relay is represented as

$$
\begin{aligned}
\mathbf{Y}_{\mathrm{i}} & =\left[\mathrm{y}_{1} \mathrm{y}_{2}\right]^{T} \\
& =\mathbf{H}_{\mathrm{i}-1 \mathrm{i}} \mathbf{P}_{\mathrm{i}-1}^{\mathrm{F}} \mathbf{S}_{\mathrm{i}-1}^{\mathrm{F}}+\mathbf{H}_{\mathrm{ii}+1} \mathbf{P}_{\mathrm{i}+1}^{\mathrm{B}} \mathbf{S}_{\mathrm{i}+1}^{\mathrm{B}}+\mathbf{S}_{\mathrm{i}}^{\mathrm{int}}+\mathbf{N}_{\mathrm{i}},
\end{aligned}
$$

here, $\mathbf{H}_{\mathrm{i}-1 \mathrm{i}} \in \mathbb{C}^{2 \times 2}$ and $\mathbf{H}_{\mathrm{ii}+1} \in \mathbb{C}^{2 \times 2}$ denote channel response matrices between $\mathrm{i}-1^{\text {th }}$ and $\mathrm{i}^{\text {th }}$ relays, between $\mathrm{i}^{\text {th }}$ and $\mathrm{i}+1^{\text {th }}$ relays, respectively. $\mathbf{N}_{\mathrm{i}} \in \sigma^{2} \mathbb{C}^{2 \times 1}$ is the noise vector with variance of $\sigma^{2}$. The signal processing at $\mathrm{i}^{\text {th }}$ relay is to cancel the interference signal $S_{\mathrm{i}}^{\text {int }}$ and to obtain the desired signal $\mathbf{S}_{\mathrm{i}+1}^{\mathrm{B}}, \mathbf{S}_{\mathrm{i}-1}^{\mathrm{F}}$.

\subsection{Performance of CICP algorithm}

Firstly, the signal processing to obtain $\mathbf{S}_{\mathrm{i}-1}^{\mathrm{F}}$ is explained. The $\boldsymbol{\Delta}_{12} \in \mathbb{C}^{2 \times 2}$ is designed to be pseudo-inverse of $\mathbf{H}_{\mathrm{i}-1 \mathrm{i}} \mathbf{P}_{\mathrm{i}-1}^{\mathrm{F}}, \boldsymbol{\Delta}_{12}=\left(\mathbf{H}_{\mathrm{i}-1 \mathrm{i}} \mathbf{P}_{\mathrm{i}-1}^{\mathrm{F}}\right)^{-1}$. We have

$$
\begin{aligned}
{\left[\begin{array}{l}
\mathrm{r}_{1} \\
\mathrm{r}_{2}
\end{array}\right] } & =\boldsymbol{\Delta}_{12}\left[\begin{array}{l}
\mathrm{y}_{1} \\
\mathrm{y}_{2}
\end{array}\right], \\
& =\mathbf{S}_{\mathrm{i}-1}^{\mathrm{F}}+\boldsymbol{\Delta}_{12}\left(\mathbf{H}_{\mathrm{ii}+1} \mathbf{P}_{\mathrm{i}+1}^{\mathrm{B}} \mathbf{S}_{\mathrm{i}+1}^{\mathrm{B}}+\mathbf{S}_{\mathrm{i}}^{\mathrm{int}}+\mathbf{N}_{\mathrm{i}}\right) .
\end{aligned}
$$

Additionally, the $\boldsymbol{\Phi}_{12,1}$ and $\boldsymbol{\Phi}_{12,2}$ are designed to be orthogonal to $\mathbf{H}_{\mathrm{i}-1 \mathrm{i}} \mathbf{P}_{\mathrm{i}-1}^{\mathrm{F}}, \boldsymbol{\Phi}_{12,2} \boldsymbol{\Phi}_{12,1} \mathbf{H}_{\mathrm{i}-1 \mathrm{i}} \mathbf{P}_{\mathrm{i}-1}^{\mathrm{F}}=\mathbf{O}$; here, $\mathbf{O}$ is the matrix whose all components are zero. Therefore,

$$
\begin{aligned}
\hat{\mathrm{y}}_{1} & =\mathrm{r}_{1}+\mathrm{r}_{11}+\mathrm{r}_{12}, \\
& =\mathrm{r}_{1}+\mathrm{q}_{11} \boldsymbol{\Phi}_{12,2} \boldsymbol{\Phi}_{12,1}\left(\mathbf{H}_{\mathrm{ii}+1} \mathbf{P}_{\mathrm{i}+1}^{\mathrm{B}} \mathbf{S}_{\mathrm{i}+1}^{\mathrm{B}}+\mathbf{S}_{\mathrm{i}}^{\text {int }}+\mathbf{N}_{\mathrm{i}}\right)\{1\} \\
& +\mathrm{q}_{12} \boldsymbol{\Phi}_{12,2} \boldsymbol{\Phi}_{12,1}\left(\mathbf{H}_{\mathrm{ii}+1} \mathbf{P}_{\mathrm{i}+1}^{\mathrm{B}} \mathbf{S}_{\mathrm{i}+1}^{\mathrm{B}}+\mathbf{S}_{\mathrm{i}}^{\text {int }}+\mathbf{N}_{\mathrm{i}}\right)\{2\} .
\end{aligned}
$$

From (3) and (4), the interference signal is cancelled, and the desired signal $\mathbf{S}_{\mathrm{i}-1}^{\mathrm{F}}\{1\}$ is obtained when

$$
\begin{aligned}
& \boldsymbol{\Delta}_{12}\left(\mathbf{H}_{\mathrm{ii}+1} \mathbf{P}_{\mathrm{i}+1}^{\mathrm{B}} \mathbf{S}_{\mathrm{i}+1}^{\mathrm{B}}+\mathbf{S}_{\mathrm{i}}^{\mathrm{int}}\right)\{1\} \\
+ & \mathrm{q}_{11} \boldsymbol{\Phi}_{12,2} \boldsymbol{\Phi}_{12,1}\left(\mathbf{H}_{\mathrm{ii}+1} \mathbf{P}_{\mathrm{i}+1}^{\mathrm{B}} \mathbf{S}_{\mathrm{i}+1}^{\mathrm{B}}+\mathbf{S}_{\mathrm{i}}^{\mathrm{int}}\right)\{1\} \\
+ & \mathrm{q}_{12} \boldsymbol{\Phi}_{12,2} \boldsymbol{\Phi}_{12,1}\left(\mathbf{H}_{\mathrm{ii}+1} \mathbf{P}_{\mathrm{i}+1}^{\mathrm{B}} \mathbf{S}_{\mathrm{i}+1}^{\mathrm{B}}+\mathbf{S}_{\mathrm{i}}^{\text {int }}\right)\{2\}=0,
\end{aligned}
$$

for any $\mathbf{S}_{\mathbf{i}+1}^{\mathrm{B}}$ and $\mathbf{S}_{\mathrm{i}}^{\text {int }}$.

Let

$$
\begin{aligned}
\boldsymbol{\Delta}_{12} & =\left[\begin{array}{ll}
\delta_{11} & \delta_{12} \\
\delta_{21} & \delta_{22}
\end{array}\right], \\
\boldsymbol{\Phi}_{12,2} \boldsymbol{\Phi}_{12,1} & =\left[\begin{array}{ll}
\phi_{11} & \phi_{12} \\
\phi_{21} & \phi_{22}
\end{array}\right], \\
\mathbf{H}_{\mathrm{ii}+1} \mathbf{P}_{\mathrm{i}+1}^{\mathrm{B}} \mathbf{S}_{\mathrm{i}+1}^{\mathrm{B}}+\mathbf{S}_{\mathrm{i}}^{\text {int }} & =\left[\begin{array}{ll}
\mathrm{s}_{11} & \mathrm{~s}_{12} \\
\mathbf{s}_{21} & \mathrm{~s}_{22}
\end{array}\right] .
\end{aligned}
$$

Hence, it leads to the following condition.

$$
\begin{aligned}
\delta_{11} \mathrm{~s}_{11}+\delta_{12} \mathrm{~s}_{21} & +\mathrm{q}_{11}\left(\phi_{11} \mathrm{~s}_{11}+\phi_{12} \mathrm{~s}_{21}\right) \\
& +\mathrm{q}_{12}\left(\phi_{21} \mathrm{~s}_{11}+\phi_{22} \mathrm{~s}_{21}\right)=0, \\
\delta_{11} \mathrm{~s}_{12}+\delta_{12} \mathrm{~s}_{22} & +\mathrm{q}_{11}\left(\phi_{11} \mathrm{~s}_{12}+\phi_{12} \mathrm{~s}_{22}\right) \\
& +\mathrm{q}_{12}\left(\phi_{21} \mathrm{~s}_{12}+\phi_{22} \mathrm{~s}_{22}\right)=0,
\end{aligned}
$$

for any $\mathrm{s}_{11}, \mathrm{~s}_{12}, \mathrm{~s}_{21}, \mathrm{~s}_{22}$. Thus,

$$
\begin{gathered}
\delta_{11}+\mathrm{q}_{11} \phi_{11}+\mathrm{q}_{12} \phi_{21}=0, \\
\delta_{12}+\mathrm{q}_{11} \phi_{12}+\mathrm{q}_{12} \phi_{22}=0,
\end{gathered}
$$

and then

$$
\begin{aligned}
& \mathrm{q}_{11}=\frac{\delta_{11} \phi_{22}-\delta_{12} \phi_{21}}{\phi_{21} \phi_{12}-\phi_{22} \phi_{11}} \\
& \mathrm{q}_{12}=\frac{\delta_{12} \phi_{11}-\delta_{11} \phi_{12}}{\phi_{21} \phi_{12}-\phi_{22} \phi_{11}} .
\end{aligned}
$$


Notice that we can always choose the $\mathbf{P}_{\mathrm{i}-1}^{\mathrm{F}}$ for which $\mathbf{H}_{\mathrm{i}-1 \mathrm{i}} \mathbf{P}_{\mathrm{i}-1}^{\mathrm{F}}$ has the inverse matrix and $\phi_{21} \phi_{12}-\phi_{22} \phi_{11} \neq 0$. As a result, the interference signal is completely cancelled from $\hat{y}_{1}$ when $q_{11}$ and $q_{12}$ satisfy (9). The interference also is cancelled from $\hat{y}_{2}$ by the same algorithm. Consequently, $\left[\hat{\mathrm{y}}_{1} \hat{\mathrm{y}}_{2}\right]^{T}=\mathbf{S}_{\mathrm{i}-1}^{\mathrm{F}}$ regardless of noise.

Similar to $\left[\hat{y}_{1} \hat{\mathrm{y}}_{2}\right],\left[\hat{\mathrm{y}}_{3} \hat{\mathrm{y}}_{4}\right]^{T}=\mathbf{S}_{\mathrm{i}+1}^{\mathrm{B}}$ under conditions that

$$
\begin{gathered}
\boldsymbol{\Delta}_{34}=\left(\mathbf{H}_{\mathrm{i} i+1} \mathbf{P}_{\mathrm{i}+1}^{\mathrm{B}}\right)^{-1}, \\
\boldsymbol{\Phi}_{34,2} \boldsymbol{\Phi}_{34,1} \mathbf{H}_{\mathrm{ii}+1} \mathbf{P}_{\mathrm{i}+1}^{\mathrm{B}}=\mathbf{O} .
\end{gathered}
$$

According to the precoding at transmitters and the signal processing at receivers, the CICP algorithm can cancel all interference signals and obtain the desired signal.

\subsection{Channel capacity of CICP algorithm}

As explained above, the received signal of the link between $\mathrm{i}-1^{\text {th }}$ relay and $\mathrm{i}^{\text {th }}$ relay is described as

$$
\left[\begin{array}{l}
\hat{\mathrm{y}}_{1} \\
\hat{\mathrm{y}}_{2}
\end{array}\right]=\mathbf{S}_{\mathrm{i}-1}^{\mathrm{F}}+\boldsymbol{\Delta}_{12} \mathbf{N}_{\mathrm{i}}+\boldsymbol{\Phi}_{12,2} \boldsymbol{\Phi}_{12,1}\left[\begin{array}{ll}
\mathrm{q}_{11} & \mathrm{q}_{12} \\
\mathrm{q}_{21} & \mathrm{q}_{22}
\end{array}\right] \mathbf{N}_{\mathrm{i}}
$$

However, as represented in the condition (5), the power of interference terms after multiplying by $\boldsymbol{\Delta}_{12}$ and after multiplying by $\boldsymbol{\Phi}_{12,2} \boldsymbol{\Phi}_{12,1}$ is the same due to normalizing all channel matrices. Therefore, $\mathrm{q}_{11}+\mathrm{q}_{12} \approx-1$ and $\mathrm{q}_{21}+$ $\mathrm{q}_{22} \approx-1$. Let $\hat{\mathbf{N}}_{12}$ denote the noise vector that included in $\left[\hat{y}_{1} \hat{y}_{2}\right]$,

$$
\hat{\mathbf{N}}_{12}=\boldsymbol{\Delta}_{12} \mathbf{N}_{\mathrm{i}}+\boldsymbol{\Phi}_{12,2} \boldsymbol{\Phi}_{12,1}\left[\begin{array}{ll}
\mathrm{q}_{11} & \mathrm{q}_{12} \\
\mathrm{q}_{21} & \mathrm{q}_{22}
\end{array}\right] \mathbf{N}_{\mathrm{i}}
$$

We assume that the noise vector is uncorrelated with another matrix and notice that all matrices which belong to $\mathbb{C}$ are normalized. Thus,

$$
\begin{aligned}
E\left[\hat{\mathbf{N}}_{12} \hat{\mathbf{N}}_{12}^{\mathcal{H}}\right] \approx & E\left[\boldsymbol{\Delta}_{\mathbf{1 2}} \mathbf{N}_{\mathrm{i}}\left(\boldsymbol{\Delta}_{\mathbf{1 2}} \mathbf{N}_{\mathrm{i}}\right)^{\mathcal{H}}\right] \\
& +E\left[\boldsymbol{\Phi}_{12,2} \boldsymbol{\Phi}_{12,1}\left[\begin{array}{ll}
\mathrm{q}_{11} & \mathrm{q}_{12} \\
\mathrm{q}_{21} & \mathrm{q}_{22}
\end{array}\right]\right. \\
& \left.\times \mathbf{N}_{\mathrm{i}}\left(\boldsymbol{\Phi}_{12,2} \boldsymbol{\Phi}_{12,1}\left[\begin{array}{ll}
\mathrm{q}_{11} & \mathrm{q}_{12} \\
\mathrm{q}_{21} & \mathrm{q}_{22}
\end{array}\right] \mathbf{N}_{\mathrm{i}}\right)^{\mathcal{H}}\right] \\
= & 2 \sigma^{2} .
\end{aligned}
$$

Let $\zeta$ denote a power of the received signal. The different transmit powers and the different distances have been discussed and optimized to obtain the highest channel capacity in $[13,14]$. In this paper, we assume that the transmit power of every transmitter and the distance between each transceiver are the same. Therefore, signal to interference plus noise ratios (SINRs) and channel capacities of all links are equal and represented as follows.
Notice that the interference is completely cancelled by the proposed CICP algorithm in the perfect CSI scheme.

$$
\begin{aligned}
\operatorname{SINR}_{\mathrm{i}} & =\frac{\zeta}{2 \sigma^{2}} \\
\mathrm{C}_{\mathrm{i}} & =2 \log _{2}\left(1+\mathrm{SINR}_{\mathrm{i}}\right) .
\end{aligned}
$$

Since the channel capacity of all links are equal, the channel capacity of system, $C$, is the same as $C_{i}$. However, the odd relay and the even relay transmit in the different time slot, therefore, the channel capacity of system becomes $\mathrm{C}=\frac{\mathrm{C}_{\mathrm{i}}}{2}$.

\section{Imperfect CSI scheme}

\subsection{Channel respond matrix of imperfect CSI}

The CICP algorithm was proposed, and the performance was analyzed in the perfect CSI scheme in the previous section. However, the perfect CSI scheme is not always practical because of channel estimation errors. In order to evaluate the proposal CICP algorithm, the estimated noisy channel respond matrix is described as

$$
\mathbf{H}_{\mathrm{i}-1 \mathrm{i}}=\rho \hat{\mathbf{H}}_{\mathrm{i}-1 \mathrm{i}}+\xi \overline{\mathbf{H}}_{\mathrm{i}-1 \mathrm{i}}
$$

here, $\overline{\mathbf{H}}_{\mathrm{i}-1 \mathrm{i}}$ denotes the estimated error channel matrix while $\hat{\mathbf{H}}_{\mathrm{i}-1 \mathrm{i}}$ is the true one; $\overline{\mathbf{H}}_{\mathrm{i}-1 \mathrm{i}}, \hat{\mathbf{H}}_{\mathrm{i}-1 \mathrm{i}} \in \mathbb{C}^{2 \times 2}$. Moreover, $\xi=\sqrt{1-\rho^{2}}$ is the measure of estimated error of noisy channel. Therefore, when the channel estimation error is present, under the assumption that $\xi \ll 1$, the Taylor expansion of pseudo inverse channel matrix is represented as follows.

$$
\begin{aligned}
\mathbf{H}_{\mathrm{i}-1 \mathrm{i}}^{-1} & =\left(\rho \hat{\mathbf{H}}_{\mathrm{i}-1 \mathrm{i}}+\xi \overline{\mathbf{H}}_{\mathrm{i}-1 \mathrm{i}}\right)^{-1} \\
& =\left(\rho \hat{\mathbf{H}}_{\mathrm{i}-1 \mathrm{i}} \hat{\mathbf{H}}_{\mathrm{i}-1 \mathrm{i}}^{-1} \hat{\mathbf{H}}_{\mathrm{i}-1 \mathrm{i}}+\xi \overline{\mathbf{H}}_{\mathrm{i}-1 \mathrm{i}} \hat{\mathbf{H}}_{\mathrm{i}-1 \mathrm{i}}^{-1} \hat{\mathbf{H}}_{\mathrm{i}-1 \mathrm{i}}\right)^{-1} \\
& =\left(\left(\rho \mathbf{I}+\xi \overline{\mathbf{H}}_{\mathrm{i}-1 \mathrm{i}} \hat{\mathbf{H}}_{\mathrm{i}-1 \mathrm{i}}^{-1}\right) \hat{\mathbf{H}}_{\mathrm{i}-1 \mathrm{i}}\right)^{-1} \\
& =\rho \hat{\mathbf{H}}_{\mathrm{i}-1 \mathrm{i}}^{-1}\left(\mathbf{I}+\frac{\xi}{\rho} \overline{\mathbf{H}}_{\mathrm{i}-1 \mathrm{i}} \hat{\mathbf{H}}_{\mathrm{i}-1 \mathrm{i}}^{-1}\right)^{-1}
\end{aligned}
$$

here, I represents $2 \times 2$ unit matrix. The Taylor expansion is applied and we have

$$
\begin{aligned}
& \left(\mathbf{I}+\frac{\xi}{\rho} \overline{\mathbf{H}}_{\mathrm{i}-1 \mathrm{i}} \hat{\mathbf{H}}_{\mathrm{i}-1 \mathrm{i}}^{-1}\right)^{-1} \\
= & \mathbf{I}-\frac{\xi}{\rho} \overline{\mathbf{H}}_{\mathrm{i}-1 \mathrm{i}} \hat{\mathbf{H}}_{\mathrm{i}-1 \mathrm{i}}^{-1}+\left(\frac{\xi}{\rho} \overline{\mathbf{H}}_{\mathrm{i}-1 \mathrm{i}} \hat{\mathbf{H}}_{\mathrm{i}-1 \mathrm{i}}^{-1}\right)^{2}-\cdots, \\
\approx & \mathbf{I}-\frac{\xi}{\rho} \overline{\mathbf{H}}_{\mathrm{i}-1 \mathrm{i}} \hat{\mathbf{H}}_{\mathrm{i}-1 \mathrm{i}}^{-1} .
\end{aligned}
$$

From (16) and (17), the pseudo inverse of estimated noisy channel matrix is obtained.

$$
\mathbf{H}_{\mathrm{i}-1 \mathrm{i}}^{-1} \approx \hat{\mathbf{H}}_{\mathrm{i}-1 \mathrm{i}}^{-1}\left(\rho \mathbf{I}+\xi \overline{\mathbf{H}}_{\mathrm{i}-1 \mathrm{i}} \hat{\mathbf{H}}_{\mathrm{i}-1 \mathrm{i}}^{-1}\right) .
$$




\subsection{CICP algorithm}

The received signal of $\mathbf{i}^{\text {th }}$ relay is rewritten by

$$
\begin{aligned}
\mathbf{Y}_{\mathrm{i}} & =\left[\mathrm{y}_{1} \mathrm{y}_{2}\right]^{T} \\
& =\hat{\mathbf{H}}_{\mathrm{i}-1 \mathrm{i}} \mathbf{P}_{\mathrm{i}-1}^{\mathrm{F}} \mathbf{S}_{\mathrm{i}-1}^{\mathrm{F}}+\hat{\mathbf{H}}_{\mathrm{ii}+1} \mathbf{P}_{\mathrm{i}+1}^{\mathrm{B}} \mathbf{S}_{\mathrm{i}+1}^{\mathrm{B}}+\mathbf{S}_{\mathrm{i}}^{\mathrm{int}}+\mathbf{N}_{\mathrm{i}} .
\end{aligned}
$$

However, due to channel estimation errors, $\boldsymbol{\Delta}_{12}=$ $\left(\mathbf{H}_{\mathrm{i}-1 \mathrm{i}} \mathbf{P}_{\mathrm{i}-1}^{\mathrm{F}}\right)^{-1}$ and then

$$
\begin{aligned}
{\left[\begin{array}{l}
\mathrm{r}_{1} \\
\mathrm{r}_{2}
\end{array}\right]=} & \boldsymbol{\Delta}_{12}\left[\begin{array}{l}
\mathrm{y}_{1} \\
\mathrm{y}_{2}
\end{array}\right] \\
= & \rho \mathbf{S}_{\mathrm{i}-1}^{\mathrm{F}}+\xi\left(\mathbf{P}_{\mathrm{i}-1}^{\mathrm{F}}\right)^{-1} \hat{\mathbf{H}}_{\mathrm{i}-1 \mathrm{i}}^{-1} \overline{\mathbf{H}}_{\mathrm{i}-1 \mathrm{i}} \mathbf{P}_{\mathrm{i}-1}^{\mathrm{F}} \mathbf{S}_{\mathrm{i}-1}^{\mathrm{F}} \\
& +\boldsymbol{\Delta}_{12}\left(\mathbf{H}_{\mathrm{i}+1} \mathbf{P}_{\mathrm{i}+1}^{\mathrm{B}} \mathbf{S}_{\mathrm{i}+1}^{\mathrm{B}}+\mathbf{S}_{\mathrm{i}}^{\mathrm{int}}+\mathbf{N}_{\mathrm{i}}\right) .
\end{aligned}
$$

Compared to the perfect CSI scheme in (3), the unavailable separation term of the desired signal, $\xi\left(P_{\mathrm{i}-1}^{\mathrm{F}}\right)^{-1} \hat{\mathbf{H}}_{\mathrm{i}-1 \mathrm{i}}^{-1} \overline{\mathbf{H}}_{\mathrm{i}-1 \mathrm{i}} \mathbf{P}_{\mathrm{i}-1}^{\mathrm{F}} \mathbf{S}_{\mathrm{i}-1}^{\mathrm{F}}$, has been added as an interference term.

On the other hand, the $\boldsymbol{\Phi}_{12,2} \boldsymbol{\Phi}_{12,1}$ is still designed to be orthogonal to $\mathbf{H}_{\mathrm{i}-1 \mathrm{i}} \mathbf{P}_{\mathrm{i}-1}^{\mathrm{F}}$ meaning $\boldsymbol{\Phi}_{12,2} \boldsymbol{\Phi}_{12,1} \mathbf{H}_{\mathrm{i}-1 \mathrm{i}} \mathbf{P}_{\mathrm{i}-1}^{\mathrm{F}}=$ O. Furthermore, the linear processing $\mathrm{q}_{11}$ and $\mathrm{q}_{12}$ are satisfied with (5). Therefore, the $\hat{y}_{1}$ is changed as follows.

$$
\begin{aligned}
\hat{\mathrm{y}}_{1}= & \mathrm{r}_{1}+\mathrm{r}_{11}+\mathrm{r}_{12}, \\
= & \rho \mathbf{S}_{\mathrm{i}-1}^{\mathrm{F}}(1)+\xi\left(\mathbf{P}_{\mathrm{i}-1}^{\mathrm{F}}\right)^{-1} \hat{\mathbf{H}}_{\mathrm{i}-1 \mathrm{i}}^{-1} \overline{\mathbf{H}}_{\mathrm{i}-1 \mathrm{i}} \mathbf{P}_{\mathrm{i}-1}^{\mathrm{F}} \mathbf{S}_{\mathrm{i}-1}^{\mathrm{F}}\{1\} \\
& -\frac{\xi}{\rho}\left[\mathrm{q}_{11} \mathrm{q}_{12}\right] \boldsymbol{\Phi}_{12,2} \mathbf{\Phi}_{12,1} \overline{\mathbf{H}}_{\mathrm{i}-1 \mathrm{i}} \mathbf{P}_{\mathrm{i}-1}^{\mathrm{F}} \mathbf{S}_{\mathrm{i}-1}^{\mathrm{F}} \\
& +\xi\left(\mathbf{P}_{\mathrm{i}-1}^{\mathrm{F}}\right)^{-1} \hat{\mathbf{H}}_{\mathrm{i}-1 \mathrm{i}}^{-1} \mathbf{H}_{\mathrm{i}-1 \mathrm{i}} \hat{\mathbf{S}}_{\mathrm{i}}^{\mathrm{int}}\{1\} \\
& -\frac{\xi^{2}}{\rho}\left(\mathbf{P}_{\mathrm{i}-1}^{\mathrm{F}}\right)^{-1} \hat{\mathbf{S}}_{\mathrm{i}}^{\mathrm{int}}\{1\} \\
& -\frac{\xi}{\rho}\left[\mathrm{q}_{11} \mathrm{q}_{12}\right] \boldsymbol{\Phi}_{12,2} \boldsymbol{\Phi}_{12,1} \overline{\mathbf{H}}_{\mathrm{i}-1 \mathrm{i}} \hat{\mathbf{S}}_{\mathrm{i}}^{\mathrm{int}} \\
& +\boldsymbol{\Delta}_{12} \mathbf{N}_{\mathrm{i}}\{1\}+\left[\mathrm{q}_{11} \mathrm{q}_{12}\right] \boldsymbol{\Phi}_{12,2} \boldsymbol{\Phi}_{12,1} \mathbf{N}_{\mathrm{i}}
\end{aligned}
$$

here, $\hat{\mathbf{S}}_{\mathrm{i}}^{\text {int }}=\mathbf{S}_{\mathrm{i}}^{\mathrm{int}}+\mathbf{H}_{\mathrm{ii}+1} \mathbf{P}_{\mathrm{i}+1}^{\mathrm{B}} \mathbf{S}_{\mathrm{i}+1}^{\mathrm{B}}$ is the total interference term for $\hat{y}_{1}$ and $\hat{y}_{2}$. As shown in (21), the first term is the desired signal, the second and third terms are the unavailable separation of desired signal, the fourth and fifth terms are the incomplete cancellation of interference, and the remaining term is the noise. Hence, the SINR is described by

$$
\operatorname{SINR}_{i}=\frac{\rho^{2} \zeta}{\xi^{2} \zeta\left(1+\frac{1}{\rho^{2}}\right)+\xi^{2} \gamma\left(1+\frac{1}{\rho^{2}}+\frac{\xi^{2}}{\rho^{2}}\right)+2 \sigma^{2}}
$$

here, $\gamma$ denote the power of $\hat{\mathbf{S}}_{i}^{\text {int }}$. The channel capacity is the same as description in the previous section.

\subsection{TDMA-based smart antenna algorithm}

Since the matched/orthogonal weight algorithm for bidirectional communications [20] requests at least three antenna elements in every relay, the TDMA-based smart antenna for one-directional communication $[13,19]$ is taken into comparison. Since two slots for one-directional communication can achieve the higher channel capacity than the larger number of slots can obtain [13], the TDMA-based smart antenna with two slots is adopted to compare with the proposed algorithm. The TDMAbased smart antenna algorithm is depicted in Fig. 2. The odd relay and the even relay alternatively transmit signals; furthermore, the signal is transmitted alternatively in forward link and backward link. The transmission of every link uses MIMO technique to obtain the spatial multiplexing.

In order to cancel the co-channel interference signal of adjacent relays, the weight is controlled as follows. An example of weight control for slot 1 is explained. The weight of \#1 is generated as an initial weight. The weight of $\# 2$ is generated to be matched to the weight of $\# 1$ to receive the desired signal from \#1. However, the weight of \#3 is designed to be orthogonal to the weight of \#2 to avoid interfering with \#2. Finally, the weight of \#4 is generated to be matched to the weight of \#3. The weight control for other slots is similar. Therefore, the relay can receive the desired signal without the interference signal from the neighbor relay. However, the interference signal from another relay, $S_{i}^{\text {int }}$, affects the performance. In slot 1 of Fig. 2, \#4 receives the interference signal from $\# 1$. The power of these interference signals is denoted by $\eta$. It means that even in the perfect CSI scheme, these interference signals are present and then the SINR of TDMA-based smart antenna algorithm is described as

$$
\operatorname{SINR}_{i}=\frac{\rho^{2} \zeta}{\eta+\sigma^{2}}
$$

In case of imperfect CSI, the received signal of $\mathrm{i}^{\text {th }}$ relay is represented as follows.

$$
\mathbf{Y}_{\mathrm{i}}=\mathbf{P}_{\mathrm{i}} \hat{\mathbf{H}}_{\mathrm{i}-1 \mathrm{i}} \mathbf{P}_{\mathrm{i}-1} \mathbf{S}_{\mathrm{i}-1}+\mathbf{P}_{\mathrm{i}} \hat{\mathbf{H}}_{\mathrm{ii}+1} \mathbf{P}_{\mathrm{i}+1} \mathbf{S}_{\mathrm{i}+1}+\mathbf{S}_{\mathrm{i}}^{\mathrm{int}}+\mathbf{N}_{\mathrm{i}}
$$

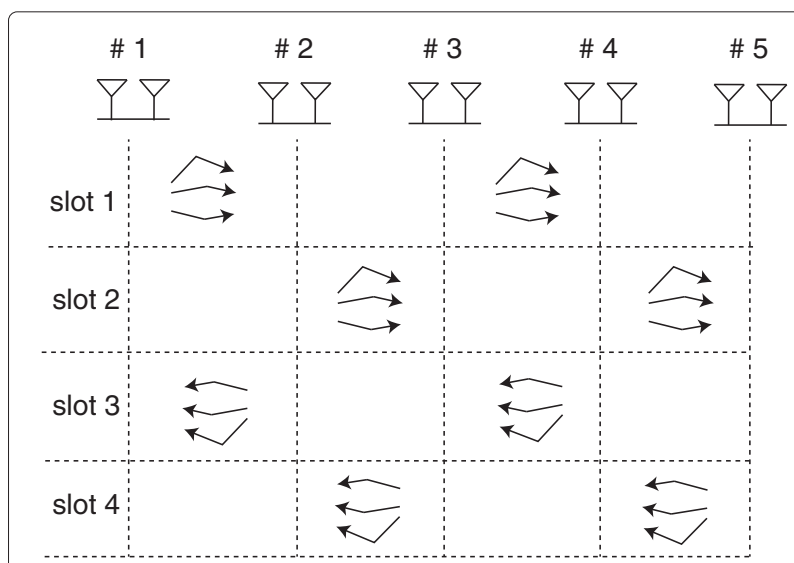

Fig. 2 TDMA-based smart antenna systems 
here,

$$
\begin{aligned}
& \mathbf{P}_{\mathrm{i}}=\left(\mathbf{H}_{\mathrm{i}-1 \mathrm{i}} \mathbf{P}_{\mathrm{i}-1}\right)^{-1}, \\
& \mathbf{P}_{\mathrm{i}} \mathbf{H}_{\mathrm{ii}+1} \mathbf{P}_{\mathrm{i}+1}=\mathbf{O}, \\
& \mathbf{H}_{\mathrm{i}-1 \mathrm{i}}=\rho \hat{\mathbf{H}}_{\mathrm{i}-1 \mathrm{i}}+\xi \overline{\mathbf{H}}_{\mathrm{i}-1 \mathrm{i}}, \\
& \mathbf{H}_{\mathrm{ii}+1}=\rho \hat{\mathbf{H}}_{\mathrm{ii}+1}+\xi \overline{\mathbf{H}}_{\mathrm{ii}+1} .
\end{aligned}
$$

Therefore, we have

$$
\begin{aligned}
\mathbf{Y}_{\mathrm{i}}= & \mathbf{P}_{\mathrm{i}-1}^{-1} \hat{\mathbf{H}}_{\mathrm{i}-1 \mathrm{i}}^{-1}\left(\rho \mathbf{I}+\xi \overline{\mathbf{H}}_{\mathrm{i}-1 \mathrm{i}} \hat{\mathbf{H}}_{\mathrm{i}-1 \mathrm{i}}^{-1}\right) \hat{\mathbf{H}}_{\mathrm{i}-1 \mathrm{i}} \mathbf{P}_{\mathrm{i}-1} \mathbf{S}_{\mathrm{i}-1} \\
& +\frac{1}{\rho} \mathbf{P}_{\mathrm{i}}\left(\mathbf{H}_{\mathrm{ii}+1}-\xi \mathbf{H}_{\mathrm{ii}+1}\right) \mathbf{P}_{\mathrm{i}+1} \mathbf{S}_{\mathrm{i}+1}+\mathbf{S}_{\mathrm{i}}^{\mathrm{int}}+\mathbf{N}_{\mathrm{i}} \\
= & \rho \mathbf{S}_{\mathrm{i}-1}+\xi \mathbf{P}_{\mathrm{i}-1}^{-1} \hat{\mathbf{H}}_{\mathrm{i}-1 \mathrm{i}}^{-1} \overline{\mathbf{H}}_{\mathrm{i}-1 \mathrm{i}} \mathbf{P}_{\mathrm{i}-1} \mathbf{S}_{\mathrm{i}-1} \\
& -\frac{\xi}{\rho} \mathbf{P}_{\mathrm{i}} \overline{\mathbf{H}}_{\mathrm{ii}+1} \mathbf{P}_{\mathrm{i}+1} \mathbf{S}_{\mathrm{i}+1}+\mathbf{S}_{\mathrm{i}}^{\mathrm{int}}+\mathbf{N}_{\mathrm{i}},
\end{aligned}
$$

and the SINR of TDMA-based smart antenna becomes

$$
\operatorname{SINR}_{i}=\frac{\rho^{2} \zeta}{\left(\xi^{2}+\frac{\xi^{2}}{\rho^{2}}\right) \zeta+\eta+\sigma^{2}} .
$$

Similar to the CICP algorithm, the unavailable separation of desired signal and the incomplete cancellation of interference signal were added. The channel capacity of each link is the same as the channel capacity of CICP algorithm.

$$
\mathrm{C}_{\mathrm{i}}=2 \log _{2}\left(1+\mathrm{SINR}_{\mathrm{i}}\right) .
$$

However, since the TDMA-based smart antenna algorithm should use four slots to complete a transmission cycle, the channel capacity of this system becomes $C=\frac{C_{i}}{4}$.

\section{Numerical evaluation}

In both the CICP and the TDMA-based smart antenna algorithm, the odd relay and the even relay alternatively transmit signals; therefore, $\mathrm{i}^{\text {th }}$ relay receives the signal from $i-1^{\text {th }}, i+1^{\text {th }}, i-3^{\text {th }}, i+3^{\text {th }}, \cdots$ relays. Since the power of received signal is assumed to be inversely proportional to the second power of distances, the power of received signal from $\mathrm{i}-3^{\text {th }}, \mathrm{i}+3^{\text {th }}$ relays is $\frac{\zeta}{9}$. In fact, $\mathrm{i}^{\text {th }}$ relay also receives the interference signal from $\mathrm{i}-5^{\text {th }}, \mathrm{i}+5^{\text {th }}$ and other relays if the number of relays is over 6; however, the power of these interference signals is greatly smaller than $\frac{\zeta}{9}$. Therefore, only the interference from $i-1^{\text {th }}, i+1^{\text {th }}$ and $i-3^{\text {th }}, i+3^{\text {th }}$ relays is taken into account. Let the number of relays in this system be 5 , the calculation result for another number of relays is similar.

Additionally, in order to evaluate the performance of proposal CICP algorithm, the signal to noise ratio (SNR) is used to calculate the channel capacity of system; here, SNR is defined as SNR $=\frac{\zeta}{\sigma^{2}}$. The channel capacity that is depicted in the following subsection is the channel capacity of system, $\mathrm{C}$.

\subsection{Perfect CSI scheme}

The comparison of channel capacities of both CICP and TDMA-based smart antenna algorithm in the perfect CSI scheme is shown in Fig. 3.

The channel capacity of both systems increases when the SNR increases. However, the channel capacity of CICP algorithm is much higher and increases more rapidly than that of the TDMA-based smart antenna algorithm. The reason is as follows. In CICP algorithm, all interference

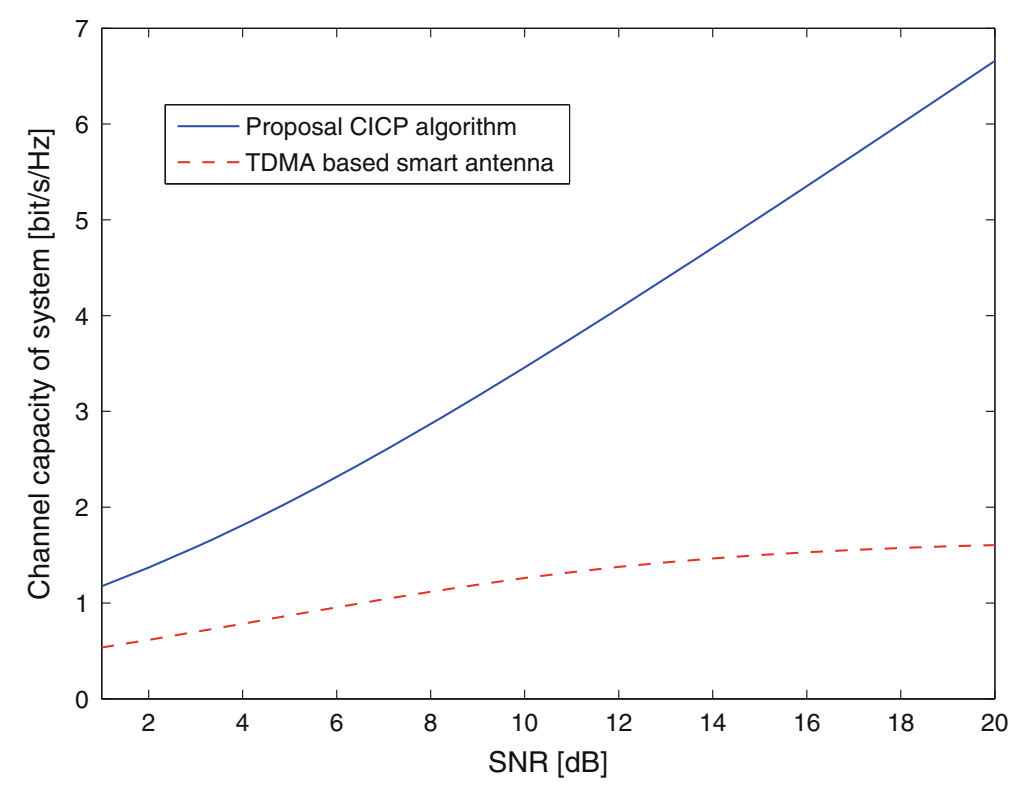

Fig. 3 The channel capacity of systems in the perfect CSI scheme 


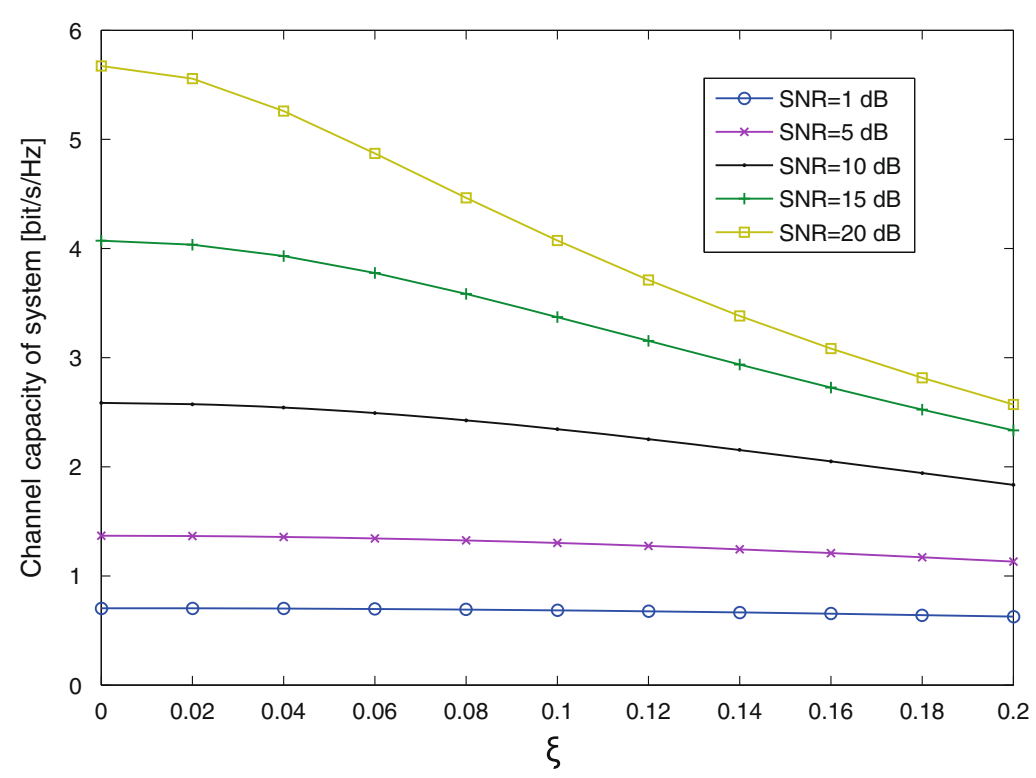

Fig. 4 The channel capacity of CICP algorithm in the imperfect CSI

signals are completely cancelled, whereas the interference signal from $\mathrm{i}-3^{\text {th }}, \mathrm{i}+3^{\text {th }}$ relays is still remained in the TDMA-based smart antenna algorithm. Therefore, the power of interference signal increases when the SNR increases. As a result, the SINR increases slowly. Additionally, the channel capacity of CICP algorithm, C, is two times smaller than the channel capacity of every link, $\mathrm{C}_{\mathrm{i}}$ (refer to Section 2.3). On the contrary, the channel capacity of TDMA-based smart antenna algorithm is four times smaller than the channel capacity of every link (refer to Section 3.3).

\subsection{Imperfect CSI scheme}

The channel capacity of CICP- and TDMA-based smart antenna algorithm is depicted in Figs. 4 and 5, respectively. The channel capacity of CICP algorithm decreases more rapidly than that of TDMA-based smart antenna algorithm when $\xi$ increases, especially for the high SNR.

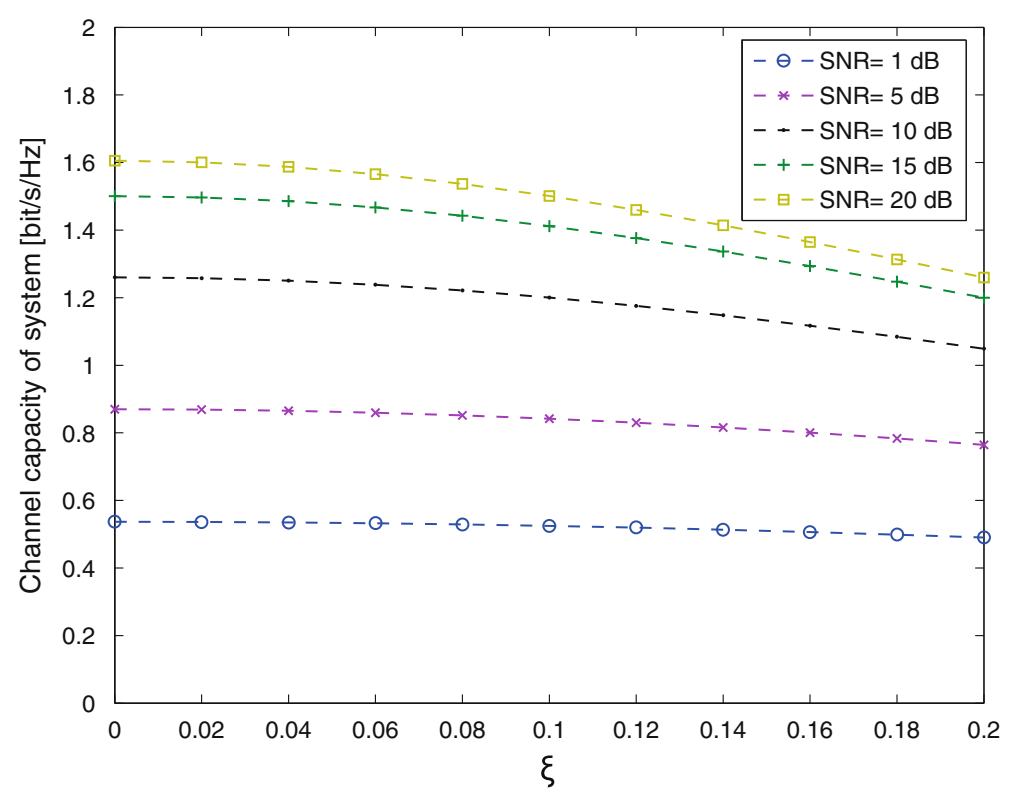

Fig. 5 The channel capacity of TDMA-based smart antenna in the imperfect CSI 
It is explained as that the power of the unavailable separation of desired signal and the incomplete cancellation of interference signal in the CICP algorithm is higher. It means that the TDMA-based smart antenna algorithm is more robust; however, when $\xi=0.2$, the channel capacity of CICP algorithm is still higher than that of the TDMA-based smart antenna algorithm.

\section{Conclusions}

In this paper, the algorithm to cancel the co-channel interference for bi-directional communication multiple hop MIMO relay systems has been proposed. The signal processing at receivers using precoding at transmitters is used to cancel the interference and obtain the desired signal. In the perfect CSI scheme, the desired signal from both forward and backward links is obtained, and all interference signals are completely cancelled. The proposal algorithm can achieve the same performance of matched/orthogonal weight algorithm. However, the matched/orthogonal weight algorithm requests at least three antenna elements at every relay, whereas the proposal algorithm can perform with only two antenna elements. The proposal algorithm is compared to the TDMA-based smart antenna algorithm that can perform with only two antenna elements. However, the TDMAbased smart antenna algorithm can just cancel the interference signal from adjacent relays, the interference signal from other relays is still remained, and only one directional communication is available. Therefore, the channel capacity of the proposal system is considerably higher than that of the TDMA-based smart antenna algorithm, especially when the SNR is high. In the imperfect CSI scheme, the channel capacity of the TDMA-based smart antenna algorithm is more robust than that of the proposal algorithm. However, the channel capacity of the proposal algorithm is still higher when the channel estimation error is large.

The proposal algorithm can obtain the high channel capacity of MHMRSs while performing with only two antenna elements. It can apply to almost all MIMO devices, even small devices, such as cell phones, vital sensors, capsule endoscopies and so on. However, in this paper, the general channel model is taken into account, and the proposal algorithm is evaluated under conditions that the SNR of all receivers is the same. The different channel models between link themselves, and approximate channel models for specific applications should be considered. In addition, the proposal algorithm is more complicated than TDMA-based smart antenna due to the signal processing at receivers. The reducing complexity also is a subject for the future research.
Received: 15 November 2014 Accepted: 5 December 2015

Published online: 18 January 2016

\section{References}

1. Yi-Sheng Su, Szu-Lin Su, Jung-Shian Li, Joint Topology-transparent scheduling and QoS routing in ad hoc networks. IEEE Trans. Veh. Technol. 63(1), 372-389 (2014)

2. SAR Zaidi, M Ghogho, DC Mclernon, A Swami, Achievable spatial throughput in multi-antenna cognitive underlay networks with multi-hop relaying. IEEE J Sel. Areas Commun. 31(8), 1543-1558 (2013)

3. Y Wei, J Heidemann, D Estrin, An energy-efficient mac protocol for wireless sensor networks. Twenty-First Annual Joint Conference of the IEEE Comput. Commun. Soc. 3, 1567-1576 (2002)

4. L Gang, B Krishnamachari, CS Raghavendra, An adaptive energy-efficient and low-latency Mac for data gathering in wireless sensor networks. 18th Int. Parallel Distrib. Process. Symp (2004). doi:10.1 109/IPDPS.2004.1303264

5. J Polastre, J Hill, D Culler, Versatile low power media access for wireless sensor networks. Second ACM Conf. Embed. Networked Sensor Syst. 2nd 95-107 (2004)

6. M Buettner, GV Yee, E Anderson, R Han, X-mac: A short preamble mac protocol for duty-cycled wireless sensor networks. Int. conf. Embed. Networked Sensor Syst. 4th, 307-320 (2006)

7. A El-Hoiydi, J-D Decotignie, Wisemac: An ultra-low power mac protocol for the multihop wireless sensor networks. Lect. Notes Comput. Sci (LNCS). 3121, 18-31 (2004)

8. P Som, A Chockalingam, Performance analysis of space shift keying in decode-and-forward multi-hop MIMO networks. IEEE Trans. Veh. Tech. 64(1), 132-146 (2014)

9. PT Hiep, NH Hoang, R Kohno, End-to-end channel capacity of multiple-hop MIMO relay system with MAC-PHY cross-layer. 9th Int. Wirel. Commun. Mobile Comput. Conf. (IWCMC), 988-992 (2013). doi:10.1109/IWCMC.2013.6583691

10. MK Arti, M Bhatnagar, MK Arti, M Bhatnagar, Maximal ratio transmission in AF MIMO relay systems over Nakagami-m Fading Channels. IEEE Trans. Veh. Technol. 64(5), 1895-1903 (2014)

11. C Symeon, MMSE filtering performance of dual-hop amplify-and-forward multiple-access channels. IEEE Wirel. Commun. Lett. 2(1), 122-125 (2013)

12. PT Hiep, K Ryuji, O Fumie, Optimizing distance, transmit power and allocation time for reliable multi-hop relay system. EURASIP J. Wirel. Commun. Netw (2012). doi:10.1186/1687-1499-2012-153

13. PT Hiep, NH Hoang, S Chika, Ryuji K, End-to-end channel capacity of $441 \mathrm{M}$ AC-PHY cross-layer multiple-hop MIMO relay system with outdated CSI. 442 EURASIP J Wirel. Commun. Netw (2013). doi:10.1186/1687-1499-2013-144

14. PT Hiep, K Ryuji, Optimizing position of repeaters in distributed MIMO repeater system for large capacity. IEICE Trans. Commun. E93-B(12), 3616-3623 (2010)

15. H Bolcskei, Nabar RU, O Oyman, AJ Paulraj, Capacity scaling laws in MIMO relay networks. IEEE Trans. Wirel. Commun. 5(6), 1433-1444 (2006)

16. M Munoz, J Vidal, A Agustin, Linear transceiver design in nonregenerative relays with channel state information. IEEE Trans. Signal Process. 55(6), 2593-2604 (2007)

17. T Le-Nam, M Juntti, M Bengtsson, B Ottersten, Weighted sum rate maximization for MIMO broadcast channels using dirty paper coding and zero-forcing methods. IEEE Trans. Commun. 61(6), 2362-2373 (2013)

18. VT Muralidharan, BS Rajan, Wireless network coding for MIMO two-way relaying. IEEE Trans. Wirel. Commun. 12(7), 3566-3577 (2013)

19. JH Winters, Smart antenna techniques and their application to wireless ad hoc networks. IEEE Wirel. Commun. 13(4), 77-83 (2006)

20. O Fumie, S Kei, MIMO spatial spectrum sharing for high efficiency mesh network. IEICE Trans. Commun. E91-B(1), 62-69 (2008) 